\title{
Investigating the Roles of Anterior Cingulate in Behavioral Variant Frontotemporal Dementia: A PET/MRI Study
}

\author{
Min Chu ${ }^{\mathrm{a}, 1}$, Li Liu ${ }^{\mathrm{a}, \mathrm{b}, 1}$, Jingjuan Wang ${ }^{\mathrm{c}}$, Lin Liu ${ }^{\mathrm{a}, \mathrm{d}}$, Yu Kong ${ }^{\mathrm{a}}$, Donglai Jing ${ }^{\mathrm{a}, \mathrm{e}}$, Kexin Xie ${ }^{\mathrm{a}}$, \\ Yue Cui ${ }^{\mathrm{a}}$, Bo Cui ${ }^{\mathrm{a}}$, Jing Zhang ${ }^{\mathrm{a}}$, Hong Ye ${ }^{\mathrm{a}}$, Junjie Li ${ }^{\mathrm{a}}$, Lin Wang ${ }^{\mathrm{a}}$, Pedro Rosa-Neto ${ }^{\mathrm{f}}$, \\ Serge Gauthier ${ }^{\mathrm{f}}$ and Liyong $\mathrm{Wu}^{\mathrm{a}, *}$ \\ ${ }^{a}$ Department of Neurology, Xuanwu Hospital, Capital Medical University, Beijing, China \\ ${ }^{\mathrm{b}}$ Department of Neurology, Shenyang Fifth People Hospital, Shenyang, China \\ ${ }^{\mathrm{c}}$ Department of Nuclear Medicine, Xuanwu Hospital, Capital Medical University, Beijing, China \\ ${ }^{\mathrm{d}}$ Department of Neurology, Second Hospital of ShanXi Medical University, Taiyuan, China \\ ${ }^{\mathrm{e}}$ Department of Neurology, Rongcheng People's Hospital, Hebei, China \\ ${ }^{\mathrm{f}}$ McGill Centre for Studies in Aging, Alzheimer's Disease Research Unit, Montreal, Canada
}

Accepted 28 September 2021

Pre-press 25 October 2021

\begin{abstract}
.
Background: The anterior cingulate cortex (ACC) seems to play an important role in behavioral deficits and executive dysfunctions in patients with behavioral variant frontotemporal dementia (bvFTD), while its specific and independent contribution requires clarification.

Objective: To identify whether ACC abnormalities in gray matter (GM) volume and standardized uptake value ratio (SUVR) images are associated with disease severity of bvFTD, by analyzing hybrid $\mathrm{T} 1$ and ${ }^{18} \mathrm{~F}$-fluorodeoxyglucose positron emission tomography $\left({ }^{18} \mathrm{~F}-\mathrm{FDG}\right.$ PET).

Methods: We enrolled 21 bvFTD patients and 21 healthy controls in the study. Each subject underwent a hybrid PET/MRI study and a standardized neuropsychologic assessment battery. GM volume and SUVR are voxel-wise calculated and compared. Then we estimate the mean value inside ACC for further partial Pearson's correlation to explore the association between GM volume/SUVR of the ACC and severity of behavioral deficit as well as executive dysfunction.

Results: ACC was shown to be involved in both atrophy and hypometabolism patterns. The partial Pearson's correlation analysis showed that the SUVR of the ACC was strongly correlated with frontal behavior inventory total score (left $r=-0.85$, right $r=-0.85, p<0.0001$ ), disinhibition subscale score (left $r=-0.72, p=0.002$; right $=-0.75, p<0.0001$ ), and apathy subscale score (left $=-0.87$, right $=-0.85, p<0.0001$ ).

Conclusion: These findings demonstrated decreased ACC activity contributes to behavioral disturbances of both apathetic and disinhibition syndromes of bvFTD, which can be sensitively detected using ${ }^{18}$ F-FDG PET.

Keywords: Anterior cingulate cortex, atrophy, ${ }^{18} \mathrm{~F}$-fluorodeoxyglucose positron emission tomography, frontotemporal dementia, hypometabolism, magnetic resonance imaging
\end{abstract}

\footnotetext{
${ }^{1}$ These authors contributed equally to this work.

*Correspondence to: Li-yong Wu, MD, PhD, Department of Neurology, Xuanwu Hospital, Capital Medical University, Beijing
}

100053, China. Tel.: +86 10 83923051; Fax: +86 1083157841 ; E-mail: wmywly@hotmail.com. 


\section{INTRODUCTION}

Behavioral variant frontotemporal dementia (bv FTD) is the most frequent subtype of frontotemporal lobe degeneration (FTLD), which primarily affects behavior regulation. It is characterized by early and prominent behavioral disinhibition, apathy or loss of sympathy, and impairment of executive function [1, 2]. bvFTD selectively impairs specific brain areas, particularly the frontal or temporal systems. In recent years, structural and metabolic research has found that the anterior cingulate cortex (ACC) is involved in bvFTD during both the prodromal and symptomatic stages, and in all subtypes of FTLD pathologies [3-14]. Also, abnormality of ACC can help discriminate bvFTD from other dementia such as Alzheimer's disease or semantic dementia on magnetic resonance imaging (MRI) with a high positive predictive value, which demonstrates its unique role in the development and progression of bvFTD $[13,15,16]$.

ACC is considered as an epicenter, known for coactivation with frontoinsula as part of a "salience network" for homeostatic behavioral guidance, that may represent the initial target in bvFTD [17]. Also, ACC is located within a significant connection of the Papez circuit, where the frontal-temporal and limbic systems link, modulating motivation, goal-directed behaviors, emotional experiences and executive functions, and a significant predictor of self-conscious emotion [16, 18-24]. Additionally, ACC had been found regulating autonomic nervous system activity in bvFTD [25]. However, the specific contributions focusing on ACC in bvFTD remain to be elucidated.

Some structural or metabolic whole-brain analysis studies in bvFTD patients have investigated the correlation between specific brain areas and behavioral dysfunctions, most of which encompassed significant findings in ACC by voxel-wise analysis, demonstrating its potential importance [6, 26-31]. However, because of lacking consistency across studies due to single neuroimage modality, limited sample size, lenient statistical thresholds, and variable measurement scales, directly integrating clinical correlation results of previous structural and metabolic research is unreliable. Hypometabolism was found more severe than gray matter (GM) atrophy in ACC, which indicates that there may be some discrepancy in the implication of clinical correlations between different modalities [12, 32]. However, the clinical correlation of ACC has not been directly analyzed by multidimensional neuropsychiatric assessments matched with the hybrid neuroimaging method in
bvFTD patients. Integrated positron emission tomography $(\mathrm{PET}) / \mathrm{MRI}$ is a recent technical innovation that allows anatomy and functional modalities to be obtained simultaneously, providing an available approach for exploring the clinical correlation [33].

In this study, patients with bvFTD underwent a hybrid PET/MRI scan and neuropsychological examinations, to explore the potential clinical contributions of ACC in bvFTD. We hypothesized that in patients with bvFTD, GM volume or standardized uptake value ratio (SUVR) changes of the ACC may have a strong correlation with the core manifestations, including behavioral disturbances and executive dysfunctions.

\section{MATERIALS AND METHODS}

\section{Ethics}

The study was conducted following the Declaration of Helsinki. The clinical protocols were approved by the ethics committee and local institutional review board of Xuanwu Hospital, Capital Medical University, China. The study was conducted following relevant guidelines and regulations for the use of human subjects in research. Written informed consent was obtained from all participants or their guardians before the start of the study.

\section{Subjects}

42 right-handed subjects are enrolled in this study, including 21 bvFTD patients and 21 healthy controls, from July 1, 2014, to December 31, 2019, in the Department of Neurology of Xuanwu Hospital. All patients were diagnosed as probable bvFTD, according to the consensus criteria published in 2011 [1]. Healthy controls were age- and sex-matched to patients and had no cognitive decline complaints, depression, or anxiety and performed within the normal range on neuropsychological tests (Mini-Mental State Examination [MMSE] score $\geq 24$ and Frontotemporal Lobar Degeneration-Clinical Dementia Rating Scale [FTLD-CDR] score of 0).

Exclusion criteria for all participants were 1) any serious neuropsychiatric disorder that could affect cognitive functioning, such as substance abuse, alcoholism, schizophrenia, tumors, or cerebrovascular disease; 2) standard contraindications for MRI examinations; and 3) absence of a reliable informant. 


\section{Neuropsychologic assessment}

Each participant underwent a standardized neuropsychologic assessment battery. Global cognitive screening was performed using the MMSE and disease severity was assessed using the FTLDCDR. Word-list memory was evaluated using Rey's Auditory-Verbal Learning Test (RAVLT). Executive function was evaluated using the Trail Making Test (TMT) and the Stroop I and II tests. Visualspatial skill was tested using the Rey complex figure test. The severity of behavioral abnormality was assessed using the Frontal Behavior Inventory (FBI), which can be separated into the negative apathy symptom subscale (first 12 items) and the positive disinhibition symptom subscale (last 12 items).

\section{PET/MRI acquisition parameters}

All images were acquired on a hybrid 3.0 T TOF PET/MRI scanner (SIGNA PET/MR, GE Healthcare, WI, USA) [34]. PET and MRI data were acquired simultaneously using a vendor-supplied 19-channel head and neck union coil. Three-dimensional (3D) T1-weighted sagittal images and ${ }^{18}$ F-FDG PET volumes were acquired during the same session after administering 3.7 MBq/kg ${ }^{18} \mathrm{~F}$-FDG for each individual.

The parameters of the T1 data were as follows: repetition time $(\mathrm{TR})=6.9 \mathrm{~ms}$, echo time $(\mathrm{TE})=2.98 \mathrm{~ms}$, flip angle $=12^{\circ}$, inversion time $=450 \mathrm{~ms}$, matrix size $=256 \times 256$, field of view $($ FOV $)=256 \times 256 \mathrm{~mm}^{2}$, slice thickness $=1 \mathrm{~mm}, 192$ sagittal slices with no gap, voxel size $=1 \times 1 \times 1 \mathrm{~mm}^{3}$, and acquisition time $=4 \mathrm{~min} 48 \mathrm{~s}$. Static ${ }^{18}$ F-FDG-PET data were acquired using the following parameters: matrix size $=192 \times$ $192, \mathrm{FOV}=350 \times 350 \mathrm{~mm}^{2}$, and pixel size $=1.82 \times$ $1.82 \times 2.78 \mathrm{~mm}^{3}$, including corrections for random coincidences, dead time, scatter, and photon attenuation. Attenuation correction was performed based on MR imaging of the brain (Atlas-based coregistration of 2-point Dixon) [35]. The default attenuation correction sequence was automatically prescribed and acquired as follows: LAVA-Flex (GE Healthcare) axial acquisition, $\mathrm{TR}=4 \mathrm{~ms}, \mathrm{TE}=1.7 \mathrm{~ms}$, slice thickness $=5.2 \mathrm{~mm}$ with a $2.6 \mathrm{~mm}$ overlap, 120 slices, pixel size $=1.95 \times 2.93 \mathrm{~mm}$, and acquisition time $=18 \mathrm{~s}$.

\section{Structural image preprocessing}

Data were preprocessed using the computational anatomy toolbox 12 (CAT 12) toolbox, (http://www. neuro.uni-jena.de/cat/), which is based on statistical parametric mapping 12 (SPM12).

First, the DICOM files were converted into nifti format, using MRICRON software (http://people. cas.sc.edu/rorden/mricron/index.html). Voxel-based morphometry (VBM) preprocessing was performed using the default settings of the CAT12 toolbox and the "East Asian Brains" ICBM template. T1weighted 3D images were segmented into GM, white matter (WM), and cerebrospinal fluid partitions. Subsequently, the GM and WM partitions of each subject in native space were high-dimensionally registered and normalized to the standard Montreal Neurological Institute (MNI) space using diffeomorphic anatomical registration through exponentiated lie algebra normalization. The images were then smoothed using an 8-mm full-width half-maximum Gaussian kernel.

\section{PET image preprocessing}

PET images were preprocessed using SPM12, implemented in MATLAB (MathWorks, Natick, Massachusetts). After normalization of the structural MRI images, the transformation parameters determined by the T1-weighted image spatial normalization were applied to the co-registered PET images for PET spatial normalization. The images were then smoothed using an isotropic Gaussian kernel with an 8-mm full-width half-maximum. Finally, PET scan intensity was normalized using a whole cerebellum reference region to create standardized uptake value ratio (SUVR) images.

\section{Voxel-based analysis and ROI analysis}

The preprocessed structural and ${ }^{18}$ F-FDG PET data were used to perform voxel-wise whole-brain comparisons between the bvFTD and control groups using Student's two-tailed $t$-test, with age, sex, and total intracranial volume as covariates. The correction threshold was set at $p<0.05$ (FWE-corrected). Then we conducted the atlas-based ROI analysis of the structural and PET images to extract the regional GM volumes and SUVR of ACC from the AAL atlas for further correlation analysis.

\section{Statistical analysis}

Statistical analyses were carried out in SPSS 22.0 (IBM, Armonk, NY, USA). Continuous data are represented as means \pm standard deviations. 
Table 1

Demographic data and neuropsychological performance in bvFTD patients $(n=21)$ and healthy controls $(n=21)$

\begin{tabular}{lccc}
\hline & bvFTD $(n=21)$ & Controls $(n=21)$ & $p$ \\
\hline Age (y) & $61.00 \pm 11.32$ & $57.95 \pm 8.00$ & 0.320 \\
Sex (male/female) & $10 / 11$ & $11 / 10$ & 0.758 \\
Years of education & $10.76 \pm 4.94$ & $11.62 \pm 3.14$ & 0.506 \\
Disease duration (y) & $2.11 \pm 0.85$ & & $<0.001$ \\
MMSE & $17.95 \pm 5.34$ & $28.73 \pm 2.09$ & \\
FTLD-CDR sum of box & $11.67 \pm 4.90$ & 0 & $<0.001$ \\
Executive function & & & $<0.001$ \\
$\quad$ TMT-A & $108.07 \pm 37.47$ & $48.47 \pm 17.66$ & 0.006 \\
$\quad$ TMT-B & $221.57 \pm 87.05$ & $82.27 \pm 45.78$ & $<004$ \\
$\quad$ Stroop I & $85.44 \pm 53.43$ & $19.13 \pm 3.14$ & $<0.001$ \\
$\quad$ Stroop II & $135.00 \pm 128.30$ & $29.07 \pm 5.80$ & $<0.001$ \\
Verbal memory & & & \\
$\quad$ RAVLT total & $14.50 \pm 10.96$ & $41.20 \pm 8.52$ & 0.004 \\
$\quad$ RAVLT recall & $1.78 \pm 2.07$ & $8.20 \pm 2.01$ & $<0.001$ \\
$\quad$ RAVLT recognition & $3.54 \pm 5.09$ & $11.73 \pm 2.49$ & $<0.001$ \\
Visuospatial skill & & & $<0.001$ \\
$\quad$ Rey complex figure test & $10.71 \pm 4.51$ & $15.00 \pm 1.41$ & \\
Behavioral features & $31.62 \pm 11.02$ & $1.57 \pm 3.04$ & \\
$\quad$ FBI total score & $13.57 \pm 5.62$ & $0.48 \pm 0.87$ & $1.10 \pm 2.23$ \\
$\quad$ FBI disinhibition score & $18.05 \pm 6.09$ & & \\
FBI apathy score & &
\end{tabular}

bvFTD, behavioral variant frontotemporal dementia; MMSE, Mini-Mental State Examination; FTLDCDR, Frontotemporal Lobar Degeneration-Clinical Dementia Rating scale; TMT, Trail Making Test; RAVLT, Rey's Auditory-Verbal Learning Test; FBI, Frontal Behavior Inventory

Dichotomous data are represented as absolute values. Group differences were tested using Student's $t$-test for continuous data and chi-square and Fisher's exact tests for categorical data. For the bvFTD group, partial Pearson's correlations between GM volume, ${ }^{18}$ F-FDG SUVR of the ACC, and neuropsychiatric assessment scores were calculated. Statistical significance was set at $p<0.05$.

\section{RESULTS}

\section{Participant demographics and neuropsychological performance}

Detailed demographic data and neuropsychological performance are summarized in Table 1. Twenty-one bvFTD patients were recruited, which included 10 men and 11 women. There were no group differences in age $(61.00 \pm 11.32$ versus $57.95 \pm 8.00, p=0.320)$, sex $(10 / 11$ versus $11 / 10$, $p=0.758)$, or years of education $(10.76 \pm 4.94$ versus $11.62 \pm 3.14, p=0.506$ ). Behavior problems were prominent in bvFTD patients, which was shown by an FBI score of $31.62 \pm 11.02$, an apathy subscale score of $18.05 \pm 6.09$, and a disinhibition subscale score of $13.57 \pm 5.62$. Patients had poor neuropsychological performance for general mental status, shown by a mean MMSE score of $<24$ and an average FTLD-CDR score of $11.67 \pm 4.90$. Moreover, executive functions were found impaired in patients: TMT-A completion time $108.07 \pm 37.47 \mathrm{~s}$, TMT-B completion time $221.57 \pm 87.05 \mathrm{~s}$, Stroop I completion time $85.44 \pm 53.43 \mathrm{~s}$, and Stroop II completion time $135.00 \pm 128.30 \mathrm{~s}$.

\section{GM atrophy and hypometabolism of the ACC}

The atrophy and hypometabolism pattern of the ACC in the bvFTD group compared with the healthy control group is shown in Fig. 1A and 1B. GM volume and SUVR of the bilateral ACC were significantly lower in patients compared with healthy controls (FWE corrected, $p<0.05$ ). Spatial coordinates and peak values of brain areas of GM atrophy and hypometabolism in bvFTD patients were shown in Table 2.

\section{Correlation analysis}

Pearson's correlation analysis of mean GM volume and SUVR values of the ACC and neuropsychological scale scores showed no significant correlations between ACC GM volume and executive function $(p>0.05)$ or behavioral deficit $(p>0.05)$. As shown 
Table 2

Spatial coordinates and peak values of brain areas showing significant GM Volume and metabolism differences between bvFTD patients and healthy controls

\begin{tabular}{|c|c|c|c|c|c|}
\hline Region & Anatomical location & Side & Cluster Size & MNI coordinate & T value \\
\hline \multicolumn{6}{|l|}{ GM atrophy } \\
\hline \multirow[t]{2}{*}{ Anterior cingulate } & Anterior cingulum & $\mathrm{R}$ & 1659 & 94518 & -12.14 \\
\hline & Anterior cingulum & $\mathrm{L}$ & 2352 & -43513 & -12.30 \\
\hline \multirow[t]{3}{*}{ Frontal lobe } & Inferior orbital frontal & $\mathrm{L}$ & 2339 & $-31.531 .5-7.5$ & -9.32 \\
\hline & Medial orbital frontal & $\mathrm{R}$ & 1096 & $1139-9$ & -12.17 \\
\hline & Middle orbital frontal & $\mathrm{R}$ & 985 & $3052.5-4.5$ & -9.08 \\
\hline \multirow[t]{3}{*}{ Temporal lobe } & Middle temporal & $\mathrm{L}$ & 4642 & $-46.5-6-13.5$ & -8.38 \\
\hline & Middle temporal & $\mathrm{R}$ & 4112 & $55-33-2$ & -8.77 \\
\hline & Superior temporal pole & $\mathrm{L}$ & 1357 & -391520 & -8.27 \\
\hline \multirow[t]{3}{*}{ Limbic lobe } & Hippocampus & $\mathrm{L}$ & 1860 & $-32-12-14$ & -12.10 \\
\hline & Insula & $\mathrm{R}$ & 3640 & 3962 & -9.07 \\
\hline & Insula & $\mathrm{L}$ & 2649 & $-30-913.5$ & -8.97 \\
\hline \multirow[t]{2}{*}{ Subcortical } & Caudate & $\mathrm{L}$ & 1492 & -121110 & -8.99 \\
\hline & Putamen & $\mathrm{R}$ & 1139 & 2853 & -9.54 \\
\hline \multicolumn{6}{|l|}{ GM hypometabolism } \\
\hline \multirow[t]{2}{*}{ Anterior cingulate } & Anterior cingulum & $\mathrm{L}$ & 1135 & -43513 & -9.24 \\
\hline & Anterior cingulum & $\mathrm{R}$ & 1040 & 22026 & -11.82 \\
\hline \multirow[t]{4}{*}{ Frontal lobe } & Middle frontal & $\mathrm{L}$ & 2644 & -343336 & -8.23 \\
\hline & Superior medial frontal & $\mathrm{L}$ & 2461 & -55031 & -11.83 \\
\hline & Superior frontal & $\mathrm{R}$ & 1763 & 167010 & -11.11 \\
\hline & Superior medial frontal & $\mathrm{R}$ & 1623 & 383435 & -8.35 \\
\hline Temporal lobe & Superior temporal pole & $\mathrm{L}$ & 2252 & $-3915-20$ & -9.07 \\
\hline Limbic lobe & Insula & $\mathrm{R}$ & 711 & $4618-10$ & -8.64 \\
\hline \multirow[t]{3}{*}{ Subcortical } & Thalamus & $\mathrm{R}$ & 736 & $6-2010$ & -8.90 \\
\hline & Putamen & $\mathrm{L}$ & 519 & -2543 & -9.26 \\
\hline & Caudate & $\mathrm{L}$ & 710 & -121110 & -10.43 \\
\hline
\end{tabular}

MNI, Montreal Neurological Institute. All regions survived the most stringent whole-brain FWE correction for multiple comparisons at $p<0.05$ (minimum cluster size 100 voxels).

\section{A GM volume bvFTD<NC}

L

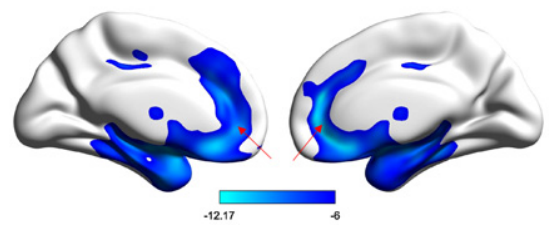

B FDG-SUVR bvFTD<NC

L

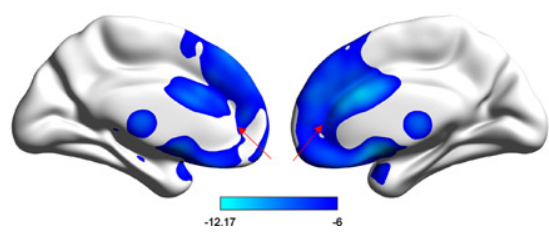

Fig. 1. GM atrophy and hypometabolism profiles. A) Regions of GM loss and B) hypometabolism in bvFTD patients. $(p<0.05$, FWE-corrected, minimum cluster size 100 voxels). T values are color-coded from cyan to blue. The red arrow indicates the location of ACC.

in Fig. 2, SUVR of the ACC was significantly correlated with the FBI total score (left $r=-0.85$, right $r=-0.85, p<0.0001$ ), disinhibition subscale score (left $r=-0.72, p=0.002$; right $=-0.75, p<0.0001$ ), and apathy subscale score (left $=-0.87$, right $=-0.85$, $p<0.0001)$. No significant correlations were found between SUVR of the ACC and scales targeting executive function $(p>0.05)$. The detailed $r$-values were shown in Table 3.

\section{DISCUSSION}

For the first time, our study explored the specific role the ACC plays in the severity of behavioral disturbances and executive dysfunction in bvFTD patients, using hybrid PET/MRI imaging. A strong significant correlation was found between SUVR of the ACC and behavioral disturbance. The findings provide in vivo evidence for the contributions of the ACC in the development of bvFTD disease dysregulation and indicate that ${ }^{18}$ F-FDG PET can offer a sensitive biomarker that reflects the severity of the disease.

Regional variability between hypometabolism and atrophy had been revealed. The voxel-wise analysis showed predominant ACC involvement in both the atrophy and hypometabolism patterns, however, hypometabolism was observed more predominant than atrophy. This phenomenon can also be observed 


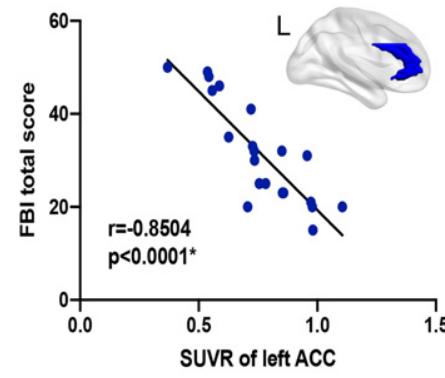

A

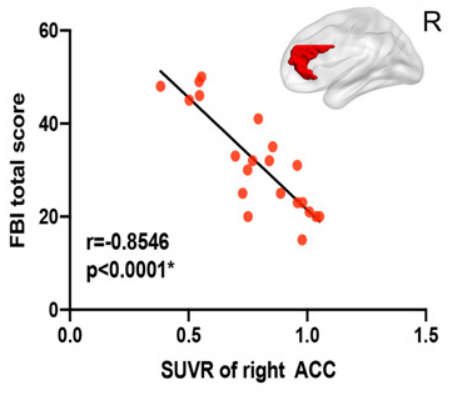

D

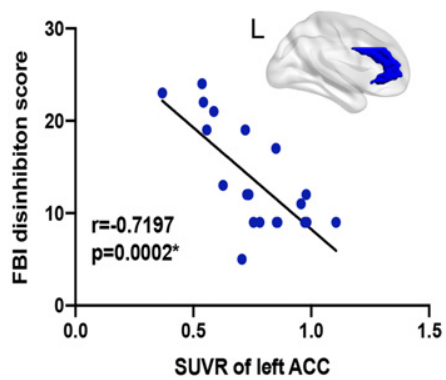

B

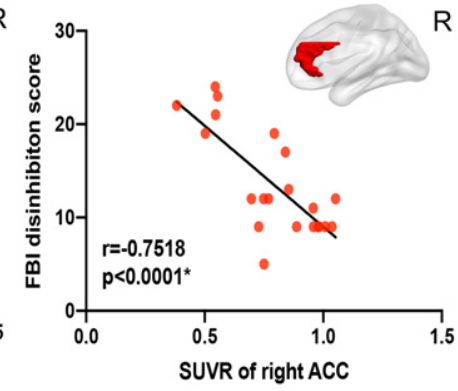

E

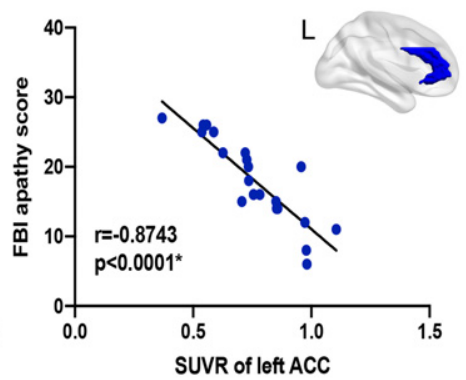

C

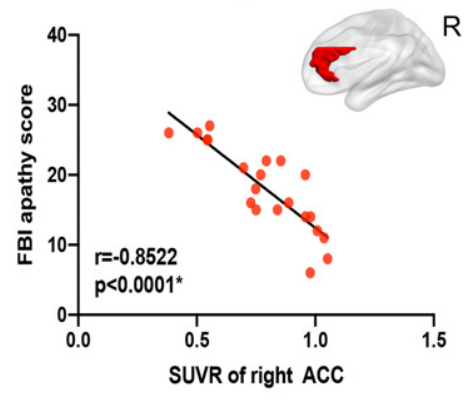

$F$

Fig. 2. Scatter plots of significant correlations in the bvFTD group. Strong correlations were found between SUVR of the left (A-C) and right ACC (D-F) and FBI total score, FBI disinhibition subscale score, as well as FBI apathy subscale score.

Table 3

Results of the partial Pearson's correlation analysis

\begin{tabular}{lccccccc}
\hline & TMT-A & TMT-B & Stroop I & Stroop II & $\begin{array}{c}\text { FBI } \\
\text { Total }\end{array}$ & $\begin{array}{c}\text { FBI } \\
\text { Apathy }\end{array}$ & $\begin{array}{c}\text { FBI } \\
\text { Disinhibition }\end{array}$ \\
\hline $\begin{array}{l}\text { GM volume } \\
\text { RACC }\end{array}$ & 0.0253 & -0.2167 & -0.5115 & -0.4185 & -0.1987 & -0.2677 & -0.0975 \\
LACC & -0.1826 & -0.4360 & -0.4648 & -0.3992 & -0.2116 & -0.3301 & -0.0542 \\
SUVR & & & & & & & \\
RACC & 0.2330 & 0.0576 & 0.5891 & 0.6436 & $-0.8546^{*}$ & $-0.7518^{*}$ & $-0.8522^{*}$ \\
LACC & -0.0380 & -0.1532 & 0.5256 & 0.5704 & $-0.8504^{*}$ & $-0.7197^{*}$ & $-0.8743^{*}$ \\
\hline
\end{tabular}

GM, gray matter; RACC, right anterior cingulum; LACC, left anterior cingulum; SUVR, standardized uptake value rate; TMT, Trail Making Test; FBI, Frontal Behavior Inventory * of statistical significance $(p<0.05)$.

in other bvFTD hybrid PET/MRI studies [12, 32]. This may be because ${ }^{18}$ F-FDG PET is more associated with functional changes, hypometabolism is not only related to GM atrophy but also proteinaceous aggregation and disposition (e.g., tau, TAR DNA-binding protein 43 , or FUS protein), which may cause genuine and more widespread functional alterations than atrophy does [36]. Another study targeting Alcoholic Korsakoff's Syndrome suggests that the hypometabolism may not only be due to local neural damage but also reflect synaptic dysfunction remote from morphological damage in other components of the functional circuit [37]. In our study, we found hemispheric asymmetry that atrophy more frequently lateralized to the left and hypometabolism to the right, which has some discrepancy with the previous study $[38,39]$. This may be due to the limited sample size with diverse disease severity and different statistical thresholds settings, which need further validation in a large cohort study.

The correlation analysis showed that SUVR of the bilateral ACC was strongly associated with behavioral disturbance. However, GM volume was not found any significant correlation. This may be attributed to the distinct pathophysiological representations of brain atrophy and cerebral hypometabolism, whereby atrophy indicates neuronal loss or reduction in the number of connections between neurons and a functional compensatory phenomenon may appear, whereas ${ }^{18}$ F-FDG PET is a 
type of molecular imaging that is based on astrocyte/neuronal glucose consumption, and ${ }^{18} \mathrm{~F}-\mathrm{FDG}$ uptake is considered a direct indicator of synaptic dysfunction [40, 41]. Hypometabolism reflects genuine functional damage because glucose is vital for the brain to participate in various critical functions, such as adenosine triphosphate production, oxidative stress management, and synthesis of neurotransmitters, neuromodulators, and structural components [41]. Coupling between function network and cognition was found stronger in presymptomatic carriers, also provide evidence that the independent and synergistic effects exist in different image modalities [42].

Some patients with bvFTD primarily present with negative symptoms, which include apathy, inertia, and loss of volition, whereas other patients predominantly present with positive symptoms, such as impulsiveness, disinhibition, and hyperactivity $[1$, 2, 43]. These two profiles have been termed "apathetic" and "disinhibited," and may overlap as the disease progresses [44]. In our study, behavioral deficits in bvFTD patients were assessed using the FBI, which is a scale specifically designed for FTD to accurately capture the severity of apathy and disinhibition, including symptoms of restlessness, irritability, and aggression as well as those related to aphasia and comprehension deficits; these provided greater sensitivity for measurements of symptoms of FTD $[43,45]$.

We found that disinhibition was associated with the bilateral ACC. Hypometabolism of the ACC is thought to impact the projection and communication of the orbitofrontal-subcortical circuit, for which its dysfunction is characterized by disinhibition symptoms, such as irritability and impulsivity [46]. In addition, other brain regions within this circuit, such as the medial prefrontal cortex, insula, and basal ganglia, were also impaired in the patients in our study. Apathy is a debilitating syndrome that is associated with numerous neurological disorders, and ACC is an anatomical correlate [47]. This is consistent with the in vivo findings of the bvFTD patients in our study. The ACC is a hub node in the ACC-subcortical circuit, which is associated with apathy [48]. Other brain areas in the ACC-subcortical circuit also showed abnormalities in our patients, such as the insula and subcortical structures, which indicated that the circuit is involved in the development of apathy in bvFTD. This requires confirmation in a further functional MRI study in a larger cohort.

We found no significant correlation between executive function and ACC both in GM volume and
SUVR, which may be due to the limited sample size and the incomprehensive assessments of executive function. However, other studies employing various methods, such as lesions, electrical stimulation, microelectrode recording, and PET, have defined ACC as a region involved in the modulation of attention in executive functions, and impairment of the ACC has been shown to result in a host of symptoms, which include inattention and executive dysfunction [22]. Fronto-striatal circuits, especially those involving the ACC, have also been shown to be related to executive functions [48, 49]. Therefore, further research in a larger bvFTD patient cohort focused on the involvement of the ACC in executive functions using assessment tools, such as the imitation sorting task, tower building, and reverse categorization, is needed to confirm whether the ACC is associated with executive function in bvFTD patients.

Moreover, ACC had been demonstrated to participate in various functions in many other studies targeting bvFTD. The integrity of gray matter areas and white matter tracts of the subcortical Papez circuit region including ACC affacting the degree of amnesia [16]. A study found that right pACC neurodegeneration played an important role in both the physiological and behavioral aspects of selfconsciousness emotion in bvFTD [24]. In addition, abnormal connectivity of ACC had been found causing integrity attenuation of salience network then lead to resting parasympathetic and sympathetic disruption [25]. All these findings suggest that $\mathrm{ACC}$ is quite an essential construct of bvFTD; however, with limited conditions, we did not perform these functional validations. Further studies are needed to validate, refine, and extend the comprehensive function of ACC.

Our study has several limitations. First, the sample was relatively small because of the challenges to enroll a large group of patients who have undergone both metabolic and structural neuroimaging. Second, a longitudinal study is warranted to observe ACC atrophy and hypometabolism and understand how they correspond to symptom progression. This will allow a deeper understanding of the ACC's role in behavioral disturbances during the natural disease course. Finally, although our bvFTD phenotypes were defined using stringent diagnostic criteria, we did not perform pathological verification. Therefore, our findings may differ slightly from those of other studies, which highlights the heterogeneity of the disease again. 


\section{CONCLUSION}

Our preliminary study provided in vivo evidence that the ACC alteration was strongly correlated with the core clinical symptoms of behavioral deficits, which demonstrates the essential role of the ACC in bvFTD disease development. Furthermore, these findings may have important implications for the use of ${ }^{18}$ F-FDG PET to establish a biomarker for the assessment of behavior alteration severity.

\section{ACKNOWLEDGMENTS}

All listed authors agree with the submission and the final manuscript has been approved by all authors. The authors wish to acknowledge Kun Yang and BinBin Nie for dedicating their time helping us in the methods.

This work was supported by grants from the National Natural Science Foundation of China [no. 81971011]; Ministry of Science and Technology of China [no. 2019YFC0118600]; and Beijing Municipal Science and Technology Committee [no.7202060].

Authors' disclosures available online (https:// www.j-alz.com/manuscript-disclosures/21-5127r2).

\section{REFERENCES}

[1] Rascovsky K, Hodges JR, Knopman D, Mendez MF, Kramer JH, Neuhaus J, Swieten J, Seelaar H, Dopper E, Hillis OAE (2011) Sensitivity of revised diagnostic criteria for the behavioural variant of frontotemporal dementia. Brain 134, 2456-2477.

[2] Neary D, Snowden J, Gustafson L, Passant U, Stuss D, Black S, Freedman M, Kertesz A, Robert P, Albert M, Boone K, Miller B, Cummings J, Benson D (1998) Frontotemporal lobar degeneration: A consensus on clinical diagnostic criteria. Neurology 51, 1546-1554.

[3] Rosen HJ, Gorno-Tempini ML, Goldman WP, Perry RJ, Miller BL (2002) Patterns of brain atrophy in frontotemporal dementia and semantic dementia. Neurology 58, 198-208.

[4] Boccardi M, Sabattoli F, Laakso MP, Testa C, Frisoni GB (2005) Frontotemporal dementia as a neural system disease. Neurobiol Aging 26, 37-44.

[5] Diehl-Schmid J, Grimmer T, Drzezga A, Bornschein S, Riemenschneider M, Förstl H, Schwaiger M, Kurz A (2007) Decline of cerebral glucose metabolism in frontotemporal dementia: A longitudinal 18F-FDG-PET-study. Neurobiol Aging 28, 42-50.

[6] Franceschi M, Anchisi D, Pelati O, Zuffi M, Matarrese M, Moresco R, Fazio F, Perani D (2005) Glucose metabolism and serotonin receptors in the frontotemporal lobe degeneration. Ann Neurol 57, 216-225.

[7] Varrone A, Pappatá S, Caracò C, Soricelli A, Milan G, Quarantelli M, Alfano B, Postiglione A, Salvatore M (2002) Voxel-based comparison of rCBF SPET images in frontotemporal dementia and Alzheimer's disease highlights the involvement of different cortical networks. Eur J Nucl Med Mol Imaging 29, 1447-1454.

[8] Nigro S, Tafuri B, Urso D, De Blasi R, Frisullo M, Barulli M, Capozzo R, Cedola A, Gigli G, Logroscino G (2021) Brain structural covariance networks in behavioral variant of frontotemporal dementia. Brain Sci 11, 192.

[9] Bueno A, de Souza L, Pinaya W, Teixeira A, de Prado L, Caramelli P, Hornberger M, Sato J (2021) Papez circuit gray matter and episodic memory in amyotrophic lateral sclerosis and behavioural variant frontotemporal dementia. Brain Imaging Behav 15, 996-1006.

[10] Clarke M, St-Onge F, Beauregard J, Bocchetta M, Todd E, Cash D, Rohrer J, Laforce R (2021) Early anterior cingulate involvement is seen in presymptomatic MAPT P301L mutation carriers. Alzheimers Res Ther 13, 42.

[11] Perry D, Brown J, Possin K, Datta S, Trujillo A, Radke A, Karydas A, Kornak J, Sias A, Rabinovici G, GornoTempini M, Boxer A, De May M, Rankin K, Sturm V, Lee S, Matthews B, Kao A, Vossel K, Tartaglia M, Miller Z, Seo S, Sidhu M, Gaus S, Nana A, Vargas J, Hwang J, Ossenkoppele R, Brown A, Huang E, Coppola G, Rosen H, Geschwind D, Trojanowski J, Grinberg L, Kramer J, Miller B, Seeley W (2017) Clinicopathological correlations in behavioural variant frontotemporal dementia. Brain 140, 3329-3345.

[12] Amanzio M, Palermo S, Stanziano M, D'Agata F, Galati A, Gentile S, Castellano G, Bartoli M, Cipriani GE, Rubino E, Fonio P, Rainero I (2021) Investigating neuroimaging correlates of early frailty in patients with behavioral variant frontotemporal dementia: A MRI and FDG-PET study. Front Aging Neurosci 13, 637796.

[13] Rabinovici GD, Seeley WW, Kim EJ, Gorno-Tempini ML, Rascovsky K, Pagliaro TA, Allison SC, Halabi C, Kramer JH, Johnson JK, Weiner MW, Forman MS, Trojanowski JQ, Dearmond SJ, Miller BL, Rosen HJ (2007) Distinct MRI atrophy patterns in autopsy-proven Alzheimer's disease and frontotemporal lobar degeneration. Am J Alzheimers Dis Other Demen 22, 474-488.

[14] Perneczky R, Diehl-Schmid J, Förstl H, Drzezga A, Kurz A (2007) Male gender is associated with greater cerebral hypometabolism in frontotemporal dementia: Evidence for sex-related cognitive reserve. Int J Geriatr Psychiatry 22, 1135-1140.

[15] Manouvelou S, Koutoulidis V, Tsougos I, Tolia M, Kyrgias G, Anyfantakis G, Moulopoulos LA, Gouliamos A, Papageorgiou S (2020) Differential diagnosis of behavioral variant and semantic variant of frontotemporal dementia using visual rating scales. Curr Med Imaging 16, 444-451.

[16] Hornberger M, Wong S, Tan R, Irish M, Piguet O, Kril J, Hodges JR, Halliday G (2012) In vivo and post-mortem memory circuit integrity in frontotemporal dementia and Alzheimer's disease. Brain 135, 3015-3025.

[17] Seeley WW (2017) Mapping neurodegenerative disease onset and progression. Cold Spring Harb Perspect Biol 9, a023622.

[18] Filippi M, Agosta F, Scola E, Canu E, Magnani G, Marcone A, Valsasina P, Caso F, Copetti M, Comi G, Cappa S, Falini A (2013) Functional network connectivity in the behavioral variant of frontotemporal dementia. Cortex 49, 23892401.

[19] Whitwell J, Josephs K, Avula R, Tosakulwong N, Weigand S, Senjem M, Vemuri P, Jones D, Gunter J, Baker M, Wszolek Z, Knopman D, Rademakers R, Petersen R, Boeve B, Jack C (2011) Altered functional connectivity in asymptomatic MAPT subjects: A comparison to bvFTD. Neurology 77, 866-874. 
[20] Zhou J, Greicius M, Gennatas ED, Growdon ME, Jang JY, Rabinovici GD, Kramer JH, Michael W, Miller BL, Seeley WW (2010) Divergent network connectivity changes in behavioural variant frontotemporal dementia and Alzheimer's disease. Brain 133(Pt 5), 1352-1367.

[21] Seeley WW, Crawford RK, Zhou J, Miller BL, Greicius M (2010) Neurodegenerative diseases target large-scale human brain networks. Neuron 62, 42-52.

[22] Vogt B, Finch D, Olson C (1992) Functional heterogeneity in cingulate cortex: The anterior executive and posterior evaluative regions. Cereb Cortex 2, 435-443.

[23] Papez J (1937) A proposed mechanism of emotion. Arch Neurol Psychiatry 38, 103-112.

[24] Sturm VE, Sollberger M, Seeley WW, Rankin KP, Ascher EA, Rosen HJ, Miller BL, Levenson RW (2013) Role of right pregenual anterior cingulate cortex in selfconscious emotional reactivity. Soc Cogn Affect Neurosci $\mathbf{8}$, 468-474.

[25] Sturm V, Brown J, Hua A, Lwi S, Zhou J, Kurth F, Eickhoff S, Rosen H, Kramer J, Miller B, Levenson R, Seeley W (2018) Network architecture underlying basal autonomic outflow: Evidence from frontotemporal dementia. $\mathrm{J} \mathrm{Neu}$ rosci 38, 8943-8955.

[26] Le Ber I, Guedj E, Gabelle A, Verpillat P, Volteau M, Thomas-Anterion C, Decousus M, Hannequin D, Véra P, Lacomblez L, Camuzat A, Didic M, Puel M, Lotterie J, Golfier V, Bernard A, Vercelletto M, Magne C, Sellal F, Namer I, Michel B, Pasquier J, Salachas F, Bochet J, Brice A, Habert M, Dubois B (2006) Demographic, neurological and behavioural characteristics and brain perfusion SPECT in frontal variant of frontotemporal dementia. Brain 129, 3051-3065.

[27] Schroeter M, Vogt B, Frisch S, Becker G, Seese A, Barthel H, Mueller K, Villringer A, Sabri O (2011) Dissociating behavioral disorders in early dementia-An FDG-PET study. Psychiatry Res 194, 235-244.

[28] McMurtray AM, Chen AK, Shapira JS, Chow TW, Mishkin F, Miller BL, Mendez MF (2006) Variations in regional SPECT hypoperfusion and clinical features in frontotemporal dementia. Neurology 66, 517-522.

[29] Peters F, Perani D, Herholz K, Holthoff V, BeuthienBaumann B, Sorbi S, Pupi A, Degueldre C, Lemaire C, Collette F, Salmon E (2006) Orbitofrontal dysfunction related to both apathy and disinhibition in frontotemporal dementia. Dement Geriatr Cogn Disord 21, 373-379.

[30] Farb NA, Grady CL, Strother S, Tang-Wai DF, Masellis M, Black S, Freedman M, Pollock BG, Campbell KL, Hasher L, Chow TW (2013) Abnormal network connectivity in frontotemporal dementia: Evidence for prefrontal isolation. Cortex 49, 1856-1873.

[31] Day GS, Farb NA, Tang-Wai DF, Masellis M, Black SE, Freedman M, Pollock BG, Chow TW (2013) Salience network resting-state activity: Prediction of frontotemporal dementia progression. JAMA Neurol 70, 1249-1253.

[32] Buhour MS, Doidy F, Laisney M, Pitel AL, de La Sayette V, Viader F, Eustache F, Desgranges B (2017) Pathophysiology of the behavioral variant of frontotemporal lobar degeneration: A study combining MRI and FDG-PET. Brain Imaging Behav 11, 240-252.

[33] Shepherd T, Nayak G (2019) Clinical use of integrated positron emission tomography-magnetic resonance imaging for dementia patients. Top Magn Reson Imaging 28, 299310 .
[34] Levin C, Maramraju S, Khalighi M, Deller T, Delso G, Jansen F (2016) Design features and mutual compatibility studies of the time-of-flight PET capable GE SIGNA PET/MR system. IEEE Trans Med Imaging 35, 1907-1914.

[35] Mainta IC, Vargas MI, Trombella S, Frisoni GB, Unschuld PG, Garibotto V (2018) Hybrid PET-MRI in Alzheimer's disease research. Methods Mol Biol 1750, 185-200.

[36] Mackenzie I, Neumann M (2016) Molecular neuropathology of frontotemporal dementia: Insights into disease mechanisms from postmortem studies. J Neurochem 138 Suppl 1, 54-70.

[37] Pitel AL, Aupée AM, Chételat G, Mézenge F, Beaunieux H, de la Sayette V, Viader F, Baron JC, Eustache F, Desgranges B (2009) Morphological and glucose metabolism abnormalities in alcoholic Korsakoff's syndrome: Group comparisons and individual analyses. PLoS One 4, e7748.

[38] Jeong Y, Cho SS, Park JM, Kang SJ, Lee JS, Kang E, Na DL, Kim SE (2005) 18F-FDG PET findings in frontotemporal dementia: An SPM analysis of 29 patients. J Nucl Med 46, 233-239.

[39] Kanda T, Ishii K, Uemura T, Miyamoto N, Yoshikawa T, Kono AK, Mori E (2008) Comparison of grey matter and metabolic reductions in frontotemporal dementia using FDG-PET and voxel-based morphometric MR studies. Eur J Nucl Med Mol Imaging 35, 2227-2234.

[40] Basu S, Hess S, Braad PN, Olsen B, Inglev S, HøilundCarlsen PF (2014) The basic principles of FDG-PET/CT imaging. PET Clinics 9, 355-370.

[41] Mukku S, Sivakumar P, Nagaraj C, Mangalore S, Harbishettar V, Varghese M (2019) Clinical utility of 18FFDG-PET/MRI brain in dementia: Preliminary experience from a geriatric clinic in South India. Asian J Psychiatry 44, 99-105.

[42] Tsvetanov K, Gazzina S, Jones P, van Swieten J, Borroni B, Sanchez-Valle R, Moreno F, Laforce R, Graff C, Synofzik M, Galimberti D, Masellis M, Tartaglia M, Finger E, Vandenberghe R, de Mendonça A, Tagliavini F, Santana I, Ducharme S, Butler C, Gerhard A, Danek A, Levin J, Otto M, Frisoni G, Ghidoni R, Sorbi S, Rohrer J, Rowe J (2021) Brain functional network integrity sustains cognitive function despite atrophy in presymptomatic genetic frontotemporal dementia. Alzheimers Dement 17, 500-514.

[43] Kertesz A, Davidson W, Fox H (1997) Frontal behavioral inventory: Diagnostic criteria for frontal lobe dementia. Can J Neurol Sci 24, 29-36.

[44] Snowden JS, Bathgate D, Varma A, Blackshaw A, Gibbons ZC, Neary D (2001) Distinct behavioural profiles in frontotemporal dementia and semantic dementia. J Neurol Neurosurg Psychiatry 70, 323-332.

[45] Boutoleau-Bretonnière C, Lebouvier $T$, Volteau C, Jaulin P, Lacomblez L, Damier P, Thomas-Anterion C, Vercelletto M (2012) Prospective evaluation of behavioral scales in the behavioral variant of frontotemporal dementia. Dement Geriatr Cogn Disord 34, 75.

[46] Cummings JL (1995) Anatomic and behavioral aspects of frontal-subcortical circuits. Ann N Y Acad Sci 769, 1-13.

[47] Le Heron C, Apps MAJ, Husain M (2018) The anatomy of apathy: A neurocognitive framework for amotivated behaviour. Neuropsychologia 118, 54-67.

[48] Bonelli RM, Cummings JL (2007) Frontal-subcortical circuitry and behavior. Dialogues Clin Neurosci 9, 141-151.

[49] Nobis L, Husain M (2018) Apathy in Alzheimer's disease. Curr Opin Behav Sci 22, 7-13. 